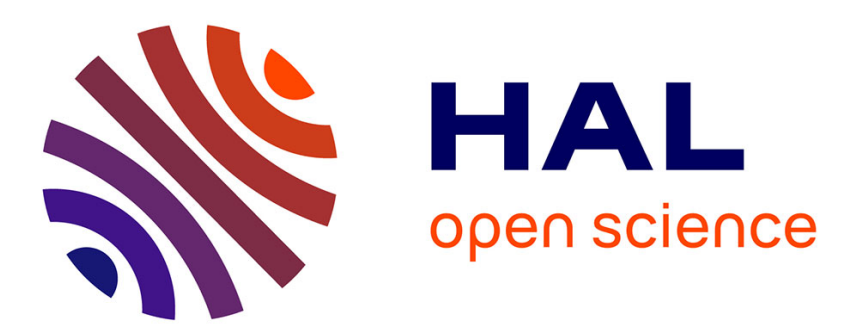

\title{
Transmission d'un filtre de masse quadrupolaire I. Etude expérimentale de la transmission d'un faisceau fin
}

\author{
J.-F. Hennequin, R.-L. Inglebert
}

\section{To cite this version:}

J.-F. Hennequin, R.-L. Inglebert. Transmission d'un filtre de masse quadrupolaire I. Etude expérimentale de la transmission d'un faisceau fin. Revue de Physique Appliquée, 1979, 14 (1), pp.275-287. 10.1051/rphysap:01979001401027500 . jpa-00244587

\section{HAL Id: jpa-00244587 https://hal.science/jpa-00244587}

Submitted on 1 Jan 1979

HAL is a multi-disciplinary open access archive for the deposit and dissemination of scientific research documents, whether they are published or not. The documents may come from teaching and research institutions in France or abroad, or from public or private research centers.
L'archive ouverte pluridisciplinaire HAL, est destinée au dépôt et à la diffusion de documents scientifiques de niveau recherche, publiés ou non, émanant des établissements d'enseignement et de recherche français ou étrangers, des laboratoires publics ou privés. 


\title{
Transmission d'un filtre de masse quadrupolaire I. Etude expérimentale de la transmission d'un faisceau fin
}

\author{
J.-F. Hennequin et R.-L. Inglebert \\ C.N.R.S., Laboratoire P.M.T.M., Université Paris-Nord, \\ avenue Jean-Baptiste-Clément, 93430 Villetaneuse, France
}

(Rę̧u le 12 juillet 1978, accepté le 9 octobre 1978)

\begin{abstract}
Résumé. - La transmission d'un filtre de masse quadrupolaire est étudiée expérimentalement au moyen d'une source d'ions alcalins $\left(\mathrm{Na}^{+}, \mathrm{Rb}^{+}\right.$et $\left.\mathrm{Cs}^{+}\right)$mobile sous vide en translation et rotation, et fournissant un étroit faisceau d'ions monocinétiques (de $20 \mathrm{à} 100 \mathrm{eV}$ ). Les résultats obtenus sont discutés et comparés aux prévisions théoriques existantes ; ils confirment le rôle essentiel du temps, mesuré en cycles HF, mis par les ions à traverser le champ d'entrée.

Abstract. - The transmission of a quadrupole mass filter is experimentally studied by means of an alkali ion source $\left(\mathrm{Na}^{+}, \mathrm{Rb}^{+}\right.$and $\left.\mathrm{Cs}^{+}\right)$movable under vacuum in translation and rotation, and giving a narrow ion beam of well-defined energy (between 20 and $100 \mathrm{eV}$ ). The results are discussed and compared with existing theoretical calculations. The time (measured in RF cycles) spent by the ions through the fringing field appears to be a prominent parameter.
\end{abstract}

1. Introduction. - Le filtre de masse à champ quadrupolaire électrique proposé par Paul et al. $[1,2]$ est largement utilisé comme analyseur de gaz résiduel, en raison surtout de sa commodité d'emploi (faible poids et balayage en masses linéaire) et de son coût modique, par comparaison aux spectromètres de masse magnétiques. La résolution en masse des analyseurs quadrupolaires usuels est relativement faible et inférieure à celle des spectromètres magnétiques; elle reste cependant bien suffisante pour l'analyse en masse des ions secondaires émis par une cible solide soumise à un bombardement ionique [3, 4], tant que les interférences entre pics de même nombre de masse sont négligeables [5]. La relative insensibilité du filtre quadrupolaire à l'énergie initiale des ions constitue alors une propriété intéressante.

Le mouvement des ions à l'intérieur d'un champ quadrupolaire a fait l'objet de nombreux calculs que l'on trouvera rassemblés dans l'ouvrage récent de Dawson [6]. Les effets de bords à l'entrée et à la sortie du quadrupôle sont difficiles à prendre en compte correctement dans ces calculs, bien que leur importance soit vite apparue tant aux expérimentateurs qu'aux théoriciens [7]. Ce n'est que récemment que Dawson [8], appliquant au filtre quadrupolaire les concepts d'espace de phase développés dans les accélérateurs circulaires et les pièges à ions quadrupolaires [9], a présenté une méthode de calcul qui lui a permis de faire intervenir de manière réaliste les champs d'extrémités [10].
La première partie de notre travail, celle qui fait l'objet du présent article, est l'étude expérimentale de la transmission d'un faisceau d'ions de faible ouverture pour différentes conditions d'introduction (position, direction et énergie des ions à leur entrée dans le quadrupôle). Ces précisions expérimentales sont nécessaires pour l'adaptation correcte d'un filtre de masse quadrupolaire dans un montage. Certains des résultats obtenus peuvent être comparés aux calculs existants que nous rappellerons au préalable de façon succincte. La deuxième partie du travail portera sur la détermination de la transmission d'un faisceau de grande ouverture à partir des résultats sur les faisceaux fins ; elle fera l'objet d'une publication ultérieure.

2. Rappels théoriques. - Nous adoptons dans tout ce qui suit les notations de Dawson [6]. Un champ quadrupolaire d'axe $\mathrm{O} z$ est approximativement réalisé en disposant quatre barres cylindriques parallèlement à $\mathrm{O} z$, à la distance $r_{0}$ de $\mathrm{O} z$ (Fig. 1), et en adoptant pour rayon des barres la valeur $1,148 r_{0}$. Entre les deux paires de barres opposées, on applique une différence de potentiel de la forme : $U-V \cos \omega t$, la fréquence $f=\omega / 2 \pi$ du terme sinusoïdal étant de l'ordre du mégahertz. Le plan $x z$ est le plan des deux barres opposées qui reçoivent la tension continue $\frac{1}{2} U$, de même signe que la charge des ions; le plan $y z$ est celui où les barres sont portées à la tension continue $-\frac{1}{2} U$, de signe contraire à la charge des ions. 


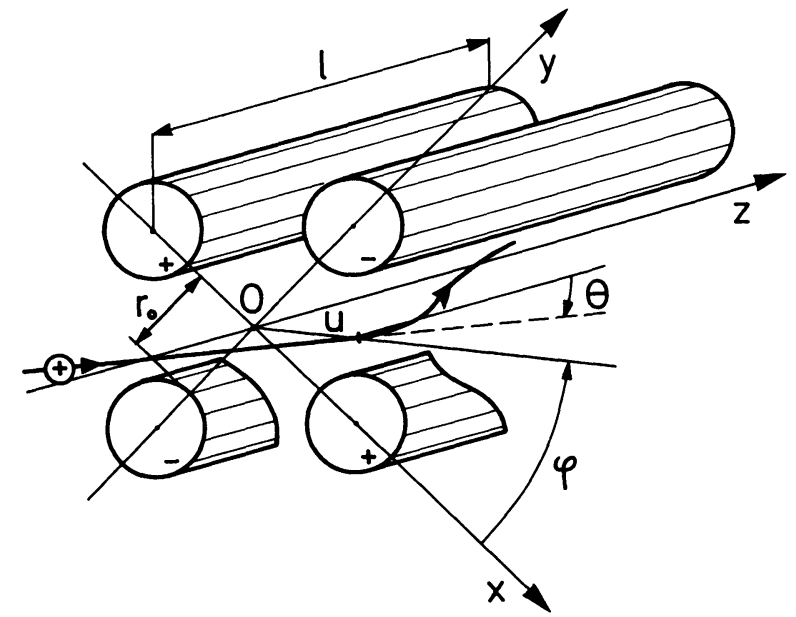

Fig. 1. - Structure géométrique et paramètres d'entrée d'un quadrupôle.

[Geometrical structure and entrance parameters of a quadrupole.]

2.1 MOUVEMENT D'UN ION DANS UN CHAMP QUADRUPOLAIRE INFINI. - A l'intérieur du champ quadrupolaire, les équations différentielles qui décrivent le mouvement d'un ion de masse $m$ et de charge $e$ sont totalement découplées dans les trois directions $x, y$ et $z$. On peut donc se limiter à l'étude des trajectoires dans les deux plans $x z$ et $y z$. Les équations en $x$ et $y$ prennent la forme canonique'de l'équation de Mathieu en introduisant les paramètres sans dimensions :

$a=4 \frac{e U}{m \omega^{2} r_{0}^{2}}$ et $q=2 \frac{e V}{m \omega^{2} r_{0}^{2}}$

tandis que l'équation en $z$ correspond à un mouvement uniforme en projection sur l'axe $\mathrm{O} z$.

Une trajectoire est dite stable lorsque l'ion ne s'écarte pas indéfiniment de l'axe $\mathrm{O} z$, mais exécute seulement des oscillations plus ou moins complexes autour de $\mathrm{O} z$. La stabilité d'une trajectoire dépend uniquement des valeurs de $a$ et $q$, donc des seules amplitudes des tensions $U$ et $V$ appliquées aux barres, et non des conditions initiales d'injection des ions.

Il est particulièrement commode d'introduire l'espace auxiliaire $(a, q)$ et d'y porter les courbes qui correspondent aux limites de stabilité en $x$ et en $y$. Pour qu'une trajectoire soit à la fois stable en $x$ et en $y$, il faut que le point de fonctionnement du quadrupôle (défini par $U$ et $V$ pour une espèce ionique donnée) se situe à l'intérieur d'une région dénommée triangle de stabilité en raison de son allure. Si le point de fonctionnement est suffisamment proche du sommet du triangle de stabilité, seuls les ions ayant un rapport $m / e$ donné pourront décrire des trajectoires stables, les ions de $m / e$ inférieur décrivant des trajectoires instables dans le plan $x z$ et ceux de $m / e$ supérieur instables dans le plan $y z$. En faisant varier les tensions $U$ et $V$ tout en maintenant constant leur rapport $U / V=a / 2 q$, on peut amener successivement à l'intérieur du triangle de stabilité les différentes valeurs du rapport $m / e$ qui correspondent à des points distincts de la droite de balayage (droite passant par l'origine et de pente $a / q$ fixée). La résolution $R=M / \Delta M$ (où $\Delta M$ est la largeur à mi-hauteur, mesurée en u.m.a., du pic de masse $M$ ) est fixée uniquement par la valeur adoptée pour le rapport $U / V$ et demeure constante sur tout le spectre de masse.

2.2 QuADRUPÔLE RÉEL. - Il faut ensuite tenir compte du fait que les barres qui créent le champ ont une longueur $l$ finie et de plus, par leur présence, limitent inéluctablement à $r_{0}$ l'écart maximal à l'axe d'une trajectoire dans les plans $x z$ et $y z$. Toutefois, l'effet des champs d'extrémités sur les trajectoires elles-mêmes ne sera abordé qu'en 2.3.

La trajectoire d'un ion dépend, non seulement de la position et de la vitesse de l'ion à l'instant initial où il pénètre dans le quadrupôle, mais aussi de la phase du champ haute-fréquence à cet instant. La transmission $T$ de l'ion est dite à $100 \%$ si l'ion peut se propager indéfiniment dans le champ quadrupolaire, quelle que soit la phase HF initiale, l'écart maximal à l'axe de sa trajectoire demeurant toujours inférieur à $r_{0}$. La transmission à $100 \%$ d'un faisceau d'ions se caractérise par des pics de masse à sommet plat (aspect trapézoïdal), dont la hauteur ne dépend pas de la résolution. Si par contre l'écart à l'axe atteint $r_{0}$ pour certaines valeurs de la phase HF, l'ion heurte une barre et est éliminé, et la transmission devient inférieure à $100 \%$. L'allure des pics de masse devient triangulaire, avec un sommet plus ou moins arrondi $[8,11]$, et leur hauteur diminue lorsque la résolution augmente.

La transmission intégrale à $100 \%$ d'un faisceau avec une résolution $R$ donnée impose des contraintes aux positions et vitesses initiales des ions [2]. Par exemple, un faisceau injecté parallèlement à l'axe $\mathrm{O} z$ doit avoir un diamètre inférieur à :

$d=2 u_{100} \simeq r_{0} \sqrt{\frac{\Delta M}{M}}=\frac{r_{0}}{\sqrt{R}}$.

De même, dans le cas d'un faisceau issu du point $O$ de l'axe situé à l'entrée du champ, la vitesse radiale des ions ne doit pas dépasser une certaine valeur limite. De leur côté, Ghosh et al. [12] ont proposé une expression empirique pour la résolution maximale possible, expression qui tient compte à la fois de la position et de la vitesse des ions à l'entrée du champ.

$\mathrm{Du}$ fait de la longueur $l$ finie des barres, les ions ne subissent qu'un nombre limité de cycles HF lors de leur propagation dans le quadrupôle, ce qui limite aussi la résolution. Diverses expériences $[2,13,14]$ montrent que le nombre $N$ de cycles nécessaires pour atteindre une résolution $R$ donnée varie sensiblement comme $\sqrt{R}$ et vaut :

$N \simeq 3,5 \sqrt{\frac{M}{\Delta M}}=3,5 \sqrt{R}$ 
lorsque $R$ est définie par la largeur à mi-hauteur des pics de masse [2] ; ceci conduit à une valeur maximale de la vitesse axiale des ions.

Il résulte de ces considérations que, pour obtenir une résolution $R$ donnée avec une transmission de $100 \%$, l'énergie $E$ d'introduction des ions ne doit pas excéder la valeur :

$E_{\max } \simeq 420 f^{2} l^{2} \frac{M}{R}$

(expression dans laquelle $E_{\max }$ est mesurée en $\mathrm{eV}$, $f$ en $\mathrm{MHz}, l$ en $\mathrm{m}$ et $M$ en u.m.a.).

De même, l'angle $\theta$ d'injection d'ions émis d'un point source $\mathrm{O}$ situé sur l'axe $\mathrm{O} z$ à l'entrée du champ est limité, pour une transmission à $100 \%$ et la résolution maximale correspondant à leur énergie d'introduction, à la valeur $\theta_{100}$ telle que :

$\operatorname{tg} \theta_{100} \simeq 3,5 \frac{r_{0}}{l}$

(expression dans laquelle $r_{0}$ et $l$ sont exprimés dans la même unité). Selon cette théorie approchée, l'angle maximal d'injection serait fixé uniquement par la géométrie du quadrupôle et ne dépendrait ni de l'énergie des ions, ni de la résolution adoptée, dès lors que l'on aurait $E \leqslant E_{\max }$.

2.3 EFFETS DES CHAMPS D'EXTRÉMITÉS. - Avant de pénétrer dans le champ quadrupolaire, l'ion injecté subit d'abord un champ de fuite qui s'étend de part et d'autre de l'extrémité des barres sur une longueur de l'ordre de $r_{0}[7,10]$. Si l'on néglige l'inévitable composante selon $\mathrm{O} z$ du champ d'entrée, tout se passe comme si les paramètres $a$ et $q$ prenaient progressivement leur valeur de réglage. On comprend alors qu'une trajectoire normalement stable en $x$ et $y$ à l'intérieur du quadrupôle reste stable en $x$, mais devient instable en $y$ lors de la traversée du champ d'entrée. Aussi ne faut-il pas s'étonner si les résultats expérimentaux sont parfois assez peu en accord avec les formules précédentes qui ne tiennent pas compte de l'existence des champs d'extrémités.

En toute rigueur, les champs de fuite introduisent une dépendance en $z$ du potentiel et les équations différentielles qui décrivent le mouvement d'un ion ne sont plus découplées. Une intégration numérique montre cependant que le couplage peut être négligé si le temps de traversée du champ d'entrée est supérieur à 0,5 cycle HF [10]. La description des trajectoires par leurs projections dans les plans $x z$ et $y z$ peut alors être conservée.

A chacun des plans $x z$ ou $y z$, on associe un espace de phase où le point figuratif du mouvement d'un ion est défini à l'instant $t$ (et donc pour une valeur de $z$ donnée) par l'écart à l'axe $u$ ( $x$ ou $y$ ) et la vitesse radiale normalisée $\dot{u}=\mathrm{d} u / \mathrm{d} \xi\left(\right.$ avec $\left.\xi=\frac{1}{2} \omega t\right)$, soit :

$\dot{u}=\frac{1}{\pi f} \sqrt{\frac{2 e E}{m}} \sin \theta$.
Dans cette description, il est par ailleurs commode de faire intervenir la vitesse axiale par le nombre $n$ de cycles HF subis par l'ion lorsqu'il parcourt une distance projetée sur $\mathrm{O} z$ égale à $r_{0}$, soit :

$n=r_{0} f \sqrt{\frac{m}{2 e E}}$

d'où la relation :

$\dot{u}=\frac{r_{0}}{n \pi} \sin \theta$.

Dans la mesure où le champ d'extrémité s'étend sur une longueur $r_{0}, n$ est une mesure du temps de traversée du champ d'entrée, le cycle HF étant pris comme unité de temps.

Un ion qui se présente à l'entrée du quadrupôle $(z=0)$, à un instant où la phase du champ HF est $\xi_{0}$, n'est transmis que si l'écart maximal à l'axe lors de son mouvement ultérieur reste inférieur à $r_{0}$. Ceci implique que le point figuratif $(u, \dot{u})$ du mouvement de l'ion à son entrée dans le champ se situe dans un certain domaine de l'espace de phase, domaine dont il est établi $[9,10]$ qu'il est limité par une ellipse centrée à l'origine et qui dépend de $n$ et de $\xi_{0}$ (et bien entendu aussi du point de fonctionnement du quadrupôle et donc de la résolution). L'intersection des ellipses correspondant à toutes les valeurs de $\xi_{0}$ définit un nouveau domaine, appelé aire d'acceptance à $100 \%$ de transmission (pour la direction $x$ ou la direction $y$ ) : à tout point figuratif intérieur à ce domaine correspond un couple $(u, \dot{u})$ de conditions d'entrée pour lequel l'ion est toujours transmis par le quadrupôle, quel que soit son instant d'arrivée.

On définit de façon similaire, pour chacune des directions $x$ et $y$, l'aire d'acceptance à $50 \%$ qui fournit les conditions limites d'injection pour lesquelles un ion a une chance sur deux d'être transmis, selon la valeur de la phase HF à l'instant d'entrée. Dawson [10] a étudié théoriquement ces aires d'acceptance à $50 \%$ et a montré qu'elles sont bien approchées par des ellipses d'équation :

$\Gamma\left(\frac{u}{r_{0}}\right)^{2}+2 A\left(\frac{u}{r_{0}}\right)\left(\frac{\dot{u}}{r_{0}}\right)+B\left(\frac{\dot{u}}{r_{0}}\right)^{2}=\varepsilon$

où le paramètre $\varepsilon$, appelé acceptance, est proportionnel à l'aire de l'ellipse. Il a calculé les paramètres $A, B, \Gamma$ et $\varepsilon$ pour différentes valeurs de la résolution $(R=55,225,600)$ et différentes valeurs du temps de séjour de l'ion dans le champ d'extrémité $(n=0,5,1,2, \ldots$ cycles HF). La connaissance des aires d'acceptance permet alors d'aborder de manière plus réaliste le montage d'un spectromètre quadrupolaire, par adaptation de l'acceptance du quadrupôle à l'émittance du système optique qui le précède [15].

3. Méthode expérimentale. - 3.1 Appareillage. - Le schéma de principe de l'appareil utilisé est 
présenté à la figure 2 . Le montage est effectué dans une enceinte en acier inoxydable en forme de croix, qu'une pompe turbomoléculaire permet d'amener rapidement à une pression de quelque $10^{-8}$ torr.

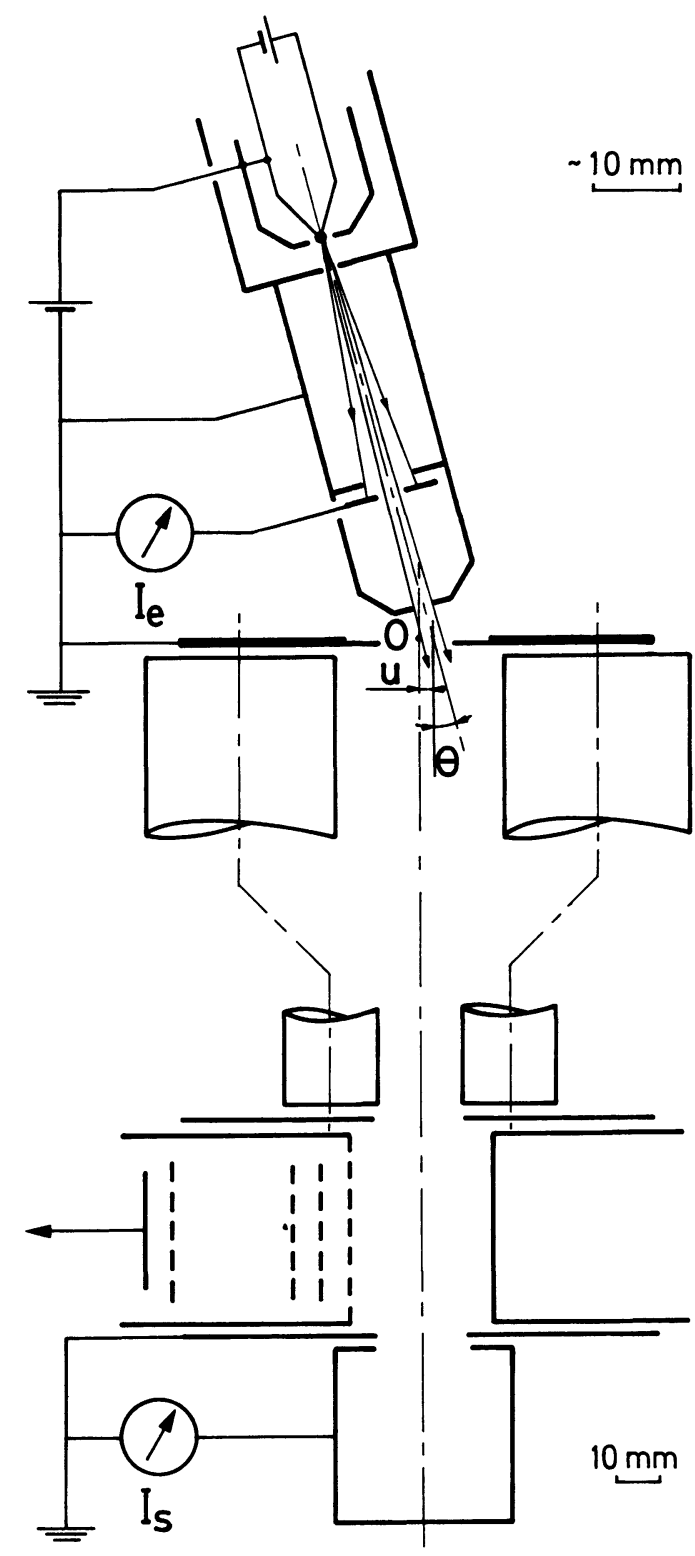

Fig. 2. - Vue schématique du dispositif expérimental. (Un grand cylindre de Faraday peut être monté à la sortie du quadrupôle, en lieu et place de l'ensemble multiplicateur d'électrons et petit cylindre ; les échelles ne sont qu'indicatives.)

[Schematic view of the experimental set up. (A large Faraday cup can be mounted at the output of the quadrupole, instead of the electron multiplier and of the small Faraday cup; the scales are indicative only).]

Les mesures ont été effectuées avec des ions alcalins : $\mathrm{Na}^{+}, \mathrm{Rb}^{+}$et $\mathrm{Cs}^{+}$fournis par une source à émission thermo-ionique [16-18]. Les ions sont émis par une petite sphère d'alumino-silicate d'environ $1 \mathrm{~mm}$ de diamètre, qu'un filament de tungstène façonné en boucle permet de porter à une température voisine de $1000^{\circ} \mathrm{C}$. Durant les premières heures de la mise en service, on observe, à côté des ions souhaités, les autres ions alcalins, surtout $\mathrm{K}^{+}$, dont l'émission diminue rapidement au fur et à mesure que ces impuretés s'éliminent. L'émission demeure ensuite raisonnablement stable sur une dizaine d'heures.

Les ions émis sont accélérés en appliquant une différence de potentiel (de 20 à $100 \mathrm{~V}$ ) entre l'extrémité négative du filament de chauffage et une électrode d'accélération maintenue au potentiel de la masse. $\mathrm{Du}$ fait de la chute ohmique de tension le long du filament et d'une différence de potentiel de contact entre le fil de tungstène et la sphère émissive, le potentiel d'émission des ions est légèrement supérieur (d'environ $1 \mathrm{~V}$ ) à la tension d'accélération. Ce point a été contrôlé en appliquant un potentiel retardateur au cylindre de Faraday destiné à collecter les ions. Nous avons simultanément constaté que la dispersion en énergie des ions n'excédait pas $0,4 \mathrm{eV}$, valeur usuelle pour une source à émission thermo-ionique [17].

Les caractéristiques optiques du faisceau d'ions sont définies par un diaphragme de $0,4 \mathrm{~mm}$ de diamètre monté au centre de l'électrode d'accélération et par un second diaphragme de $0,2 \mathrm{~mm}$ disposé le plus près possible de l'entrée du quadrupôle; la demi-ouverture du faisceau est ainsi fixée à $4,2 \times 10^{-3} \mathrm{rad}$ (environ 15'). L'intensité du courant ionique dans le faisceau passe de quelque $10^{-14} \mathrm{~A}$ pour une tension d'accélération de $20 \mathrm{~V}$ à $10^{-12} \mathrm{~A}$ pour $100 \mathrm{~V}$. Le courant ionique $I_{\mathrm{e}}$ entrant dans le quadrupôle est contrôlé en cours d'expérience en recueillant, sur un diaphragme isolé intermédiaire, une fraction définie du faisceau d'ions après accélération ; l'intensité du courant collecté par ce diaphragme est proportionnelle à l'intensité $I_{\mathrm{e}}$ dans tout le domaine d'énergies utilisées.

Le grand intérêt d'une source à ions alcalins est de ne pas nécessiter un pompage auxiliaire et donc de pouvoir assez facilement être déplacée sous vide. Le support de source, monté sur la bride supérieure de l'enceinte, permet les mouvements suivants :

- translation de $\pm 2 \mathrm{~mm}$ le long de l'axe $\mathrm{O} z$ (axe du quadrupôle), déplaçant le point source par rapport au trou d'entrée;

- translations de $\pm 2 \mathrm{~mm}$ selon deux axes perpendiculaires, contenus dans un plan perpendiculaire à $\mathrm{O} z$ (déplacement $u$ ) ;

- inclinaison de $-2^{\circ}$ à $+40^{\circ}$ de l'axe de la source d'ions par rapport à l'axe du quadrupôle (angle d'injection $\theta$ ).

De plus, comme on peut aussi changer l'orientation mutuelle des brides de fixation du support de source et du quadrupôle, il est possible de modifier, après ouverture de l'appareil, la disposition du plan d'inclinaison de la source par rapport au plan $x z$ du quadrupôle. On définit ainsi un angle azimutal $\varphi$ (Fig. 1) qui, dans ce montage, peut prendre les valeurs $0^{\circ}$ (plan $x z$ ), $18^{\circ}, 36^{\circ}, 54^{\circ}, 72^{\circ}$ et $90^{\circ}$ (plan $\left.y z\right)$. En outre, sans ouvrir l'appareil, on peut échanger les plans $x z$ et $y z$ par 
simple inversion de polarité de la tension continue $U$ appliquée aux barres du quadrupôle.

Le filtre quadrupolaire, monté sur la bride inférieure de l'enceinte, est un modèle du commerce (RIBER type $Q 156$ ) dont les caractéristiques sont les suivantes :

- rayon du champ : $r_{0}=6,7 \mathrm{~mm}$;

- longueur des barres : $l=250 \mathrm{~mm}$;

- fréquence du champ HF : $f=2 \mathrm{MHz}$.

Dans ces conditions, pour une transmission à $100 \%$ et sans tenir compte des champs d'extrémités, l'angle maximal d'injection des ions à partir d'un point source $O$ à l'entrée du champ serait : $\theta_{100} \simeq 5^{\circ} 20^{\prime}(3)$, tandis que, dans le cas d'ions injectés parallèlement à l'axe $\mathrm{O} z$, le diamètre du faisceau devrait être inférieur à $0,9 \mathrm{~mm}$ pour une résolution de 55 , et à $0,45 \mathrm{~mm}$ pour une résolution de 225 (1). Le faisceau d'ions à la sortie de la source présente donc une largeur et une ouverture suffisamment faibles, et on pourra étudier les variations de la transmission du quadrupôle en fonction de l'écart à l'axe à $\pm 0,1 \mathrm{~mm}$ près, et en fonction de l'angle d'injection à $\pm 15^{\prime}$ près (les déplacements mécaniques sont mesurés avec une précision supérieure).

Le trou d'entrée dans le quadrupôle, de diamètre $4 \mathrm{~mm}$, est percé dans une électrode de $1 \mathrm{~mm}$ d'épaisseur disposée à $1 \mathrm{~mm}$ de l'extrémité des barres et portée au potentiel de l'axe du champ quadrupolaire. Il paraît raisonnable d'estimer que le champ électrique commence à prendre une valeur appréciable au niveau de l'entrée du trou (point $\mathrm{O}$ de la figure 2, pris comme origine des abscisses $z$ ), et qu'il a sensiblement atteint sa valeur limite à l'abscisse $z=r_{0}[10]$.

Le tableau I rassemble les différentes conditions expérimentales; nature et énergie des ions utilisés (et la vitesse axiale correspondante, mesurée en nombre $n$ de cycles HF sur la longueur $r_{0}$ et donnée par la formule (5)) et résolutions adoptées. Y figurent aussi les valeurs limites théoriques, pour la résolution $R$, de l'énergie maximale $E_{\max }$ (2) et du temps de séjour minimal dans le champ de fuite soit :

$n_{\min }=3,5 \frac{r_{0}}{l} \sqrt{R}=0,094 \sqrt{R}$.

3.2 Mesure de la transmission. - Les ions qui sont transmis par le filtre quittent le champ quadrupolaire par un trou de diamètre $2 r_{0}(13,4 \mathrm{~mm})$. La mesure de l'intensité $I_{\mathrm{s}}$ du faisceau ionique sortant permet d'en déduire la transmission $T=I_{\mathrm{s}} / I_{\mathrm{e}}$ du quadrupôle pour les conditions d'injection choisies. Par des mesures répétées à différentes époques, on peut estimer à $\pm 5 \%$ l'erreur absolue sur la mesure de la transmission.

Le système usuel de collection des ions comporte un multiplicateur d'électrons monté en position latérale dans un plan bissecteur des plans $x z$ et $y z$ et un petit cylindre de Faraday de diamètre d'entrée $20 \mathrm{~mm}$ disposé à $60 \mathrm{~mm}$ de la sortie du quadrupôle (Fig. 2). Le champ électrique transversal qu'on doit appliquer entre la première dynode du multiplicateur et l'électrode opposée, assure une bonne collection des ions sortants, y compris de ceux dont la trajectoire est notablement écartée de l'axe ou très inclinée sur l'axe, tant que leur énergie n'est pas trop élevée (inférieure à $100 \mathrm{eV}$ ). Ce n'est pas le cas du petit cylindre de Faraday dont l'angle de collection est limité à $10^{\circ}$ environ. Il nous a donc semblé utile de comparer les résultats obtenus avec le multiplicateur (et accessoirement avec le petit cylindre de Faraday) avec ceux fournis par un grand cylindre de Faraday, de diamètre d'entrée $16 \mathrm{~mm}$, disposé immédiatement à la sortie du quadrupôle. Ce cylindre permet de recueillir le quasi-totalité des ions sortants, mais il ne peut être utilisé que si le courant $I_{\mathrm{s}}$ atteint au moins $10^{-13} \mathrm{~A}$.

Ces expériences comparatives ont été effectuées avec des ions $\mathrm{Rb}^{+}$de $81 \mathrm{eV}(n=1$ cycle HF) et pour la résolution $R=55$. Un diaphragme de $0,75 \mathrm{~mm}$ a été monté à la sortie de la source d'ions pour disposer d'un courant ionique suffisamment élevé. Aussi les courbes de transmission obtenues n'ont-elles pas la précision de celles présentées plus loin, obtenues avec le diaphragme normal de $0,2 \mathrm{~mm}$.

La figure $3 a$ présente les variations de la transmission pour une injection parallèle à l'axe $\mathrm{O} z$, avec déplacement du faisceau, soit dans le plan $x z$ (à gauche), soit dans le plan $y z$ (à droite). A la figure $3 b$, le faisceau est issu du point $O$ à l'entrée du quadrupôle et incliné par rapport à l'axe de l'angle $\theta$, soit dans le plan $x z$ (à gauche), soit dans le plan $y z$ (à droite). On constate sur ces figures que le grand cylindre de Faraday et le multiplicateur conduisent à des résultats tout à fait comparables tant que la transmission est assez élevée (supérieure à $30 \%$ ), alors qu'avec le petit cylindre le courant recueilli décroît bien plus rapidement, en particulier dans le plan $x z$. Ceci correspond au fait que, sous l'action du champ de fuite à la sortie, les tra-

Tableau I. - Nature et énergie des ions utilisés; résolutions adoptées

[Nature and energy of the used ions; chosen resolutions]

$\begin{array}{lccccccccc}\text { Nature des ions } & \mathrm{Na}^{+} & \mathrm{Na}^{+} & \mathrm{Na}^{+} & \mathrm{Rb}^{+} & \mathrm{Rb}^{+} & \mathrm{Rb}^{+} & \mathrm{Rb}^{+} & \mathrm{Cs}^{+} & \mathrm{Cs}^{+} \\ \text {Energie (eV) } & 100 & 100 & 22 & 81 & 81 & 20 & 20 & 32 & 32 \\ \left.n \text { (cycles HF } / r_{0}\right) & 0,5 & 0,5 & 1 & 1 & 1 & 2 & 2 & 2 & 2 \\ \text { Résolution } R & 20 & 55 & 55 & 55 & 225 & 55 & 225 & 55 & 225 \\ E_{\max }(\mathrm{eV} ;(2)) & 121 & 44 & 44 & 162 & 40 & 162 & 40 & 254 & 62 \\ n_{\min }(6) & 0,4 & 0,7 & 0,7 & 0,7 & 1,4 & 0,7 & 1,4 & 0,7 & 1,4\end{array}$




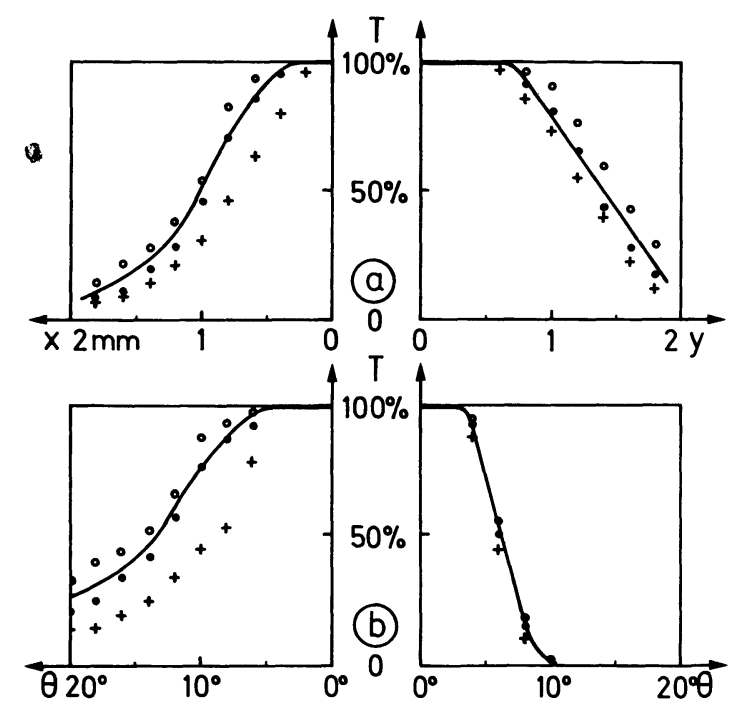

Fig. 3. - Influence de la méthode de mesure sur les variations de la transmission pour $n=1$ cycle HF et $R=55: a$ ) en fonction de la distance à l'axe pour une introduction parallèle à l'axe $; b$ ) en fonction de l'inclinaison sur l'axe pour une introduction à partir du point $\mathrm{O}$. (Ions $\mathrm{Rb}^{+}$de $81 \mathrm{eV}$. Diaphragme de sortie de source : $0,75 \mathrm{~mm}$. A gauche : plan $x z$; à droite : plan $y z$. Mesure : $:$ multiplicateur d'électrons; + : petit cylindre de Faraday; $\mathrm{O}:$ grand cylindre de Faraday.)

[Effect of the measurement method on the variations of the transmission for $n=1 \mathrm{RF}$ cycle and $R=55: a)$ versus the distance from the axis for an introduction parallel to the axis; $b$ ) versus the input

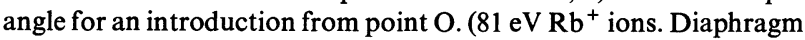
of source output : $0.75 \mathrm{~mm}$. Left : $x z$ plane ; right : $y z$ plane. Method of measurement : $\bullet$ : electron multiplier ; + : small Faraday cup ; $\mathrm{O}$ : large Faraday cup).]

jectoires ioniques s'écartent plus rapidement de l'axe dans la direction $x$ que dans la direction $y$ [7].

La plupart des mesures de transmission présentées ont été effectuées avec le multiplicateur d'électrons, le grand cylindre de Faraday ne conduisant à des courants mesurables avec une précision acceptable qu'avec les ions $\mathrm{Na}^{+}$d'une énergie supérieure à $50 \mathrm{eV}$. Les transmissions inférieures à $30 \%$ peuvent être notablement sous-estimées, mais il faut admettre qu'elles ne correspondent pas à un mode de fonctionnement bien intéressant pour un filtre de masse !

3.3 Problèmes De Mesures. - Un soin particulier a été apporté lors du montage à la détermination des caractéristiques géométriques de l'étroit faisceau d'ions entrant dans le filtre quadrupolaire. Les plans des deux brides de fixation, inférieure et supérieure, étant parallèles à mieux de $10^{\prime}$, on joue sur l'écrasement des joints de cuivre du quadrupôle pour amener le point $\mathrm{O}$ de l'axe du filtre sur l'axe de la bride de montage du support de source (contrôle à $\pm 0,05 \mathrm{~mm}$ près par mesures directes); les directions de l'axe $\mathrm{O} z$ du filtre et de l'axe de la source en position $\theta=0$ coïncident alors à $\pm 15^{\prime}$ près.

La bride du support de source étant équipée d'un joint viton et d'une portée de centrage, sa position par rapport au quadrupôle est parfaitement définie. On peut donc, par des mesures mécaniques effectuées avant montage sur l'enceinte, faire les zéros sur les trois axes de coordonnées, c'est-à-dire trouver les réglages en $x, y$ et $z$ qui amèneront à faire passer par le point $\mathrm{O}$, au montage, l'axe de la source en position $\theta=0$ (réglages $x$ et $y$, à $\pm 0,1 \mathrm{~mm}$ près) et, en cours de manipulation, l'axe d'inclinaison de la source d'ions (réglage $z$, à $\pm 0,2 \mathrm{~mm}$ près). Un contrôle des réglages des zéros en $x$ et $y$ est d'ailleurs possible en cours d'expérience, car la transmission d'un faisceau d'ions injectés parallèlement à l'axe doit nécessairement varier de façon symétrique de part et d'autre de l'axe.

Une autre difficulté est la détermination précise du point de fonctionnement du quadrupôle. Nous avons opéré en introduisant le faisceau d'ions selon l'axe et en modifiant le rapport $U / V$ (en jouant sur le réglage résolution) de manière à obtenir le pic de masse avec la largeur à mi-hauteur correspondant à la résolution choisie : 20, 55 ou 225 (mesure effectuée sur l'écran de l'oscilloscope).

Le profil du pic de masse se dégrade quelque peu quand le faisceau est écarté de l'axe. Un pic parasite apparaît sur le flanc hautes-masses [19], mais la résolution mesurée à mi-hauteur n'est guère affectée tant que l'on reste en régime trapézoïdal. La disposition latérale du multiplicateur joue sans doute aussi un rôle dans l'aspect du pic [20]. En fait, la déformation du pic reste modérée avec le grand cylindre de Faraday et le multiplicateur (étant entendu que les tensions de déflexion des ions sont correctement réglées); elle devient importante pour les ions rapides avec le petit cylindre de Faraday où, dans les situations extrêmes, on en arrive à observer deux autres pics parasites. L'incapacité du petit cylindre à collecter les ions sortants très écartés de l'axe est évidemment à l'origine de cette différence de comportement. Ces profils aberrants ne s'observent pas en utilisation normale du quadrupôle, avec des faisceaux d'ions lents, largement ouverts et correctement centrés, du fait d'une moyenne sur toutes les directions d'injection des ions.

4. Résultats expérimentaux. - 4.1 AIRES D'ACCEPTANCE. - Les aires d'acceptance pour la transmission $50 \%$ ont été déterminées pour chacune des directions $x$ ou $y$, et comparées aux prévisions théoriques de Dawson [10] pour $n=0,5,1$ et 2 cycles HF et pour $R=55$ et 225 . L'ensemble ayant fait l'objet d'une publication préliminaire [21], nous ne présentons ici, à titre d'exemple, que les résultats obtenus à $n=2$ cycles avec des ions $\mathrm{Rb}^{+}$de $20 \mathrm{eV}$ et des ions $\mathrm{Cs}^{+}$ de $32 \mathrm{eV}$. La figure 4 correspond à la résolution 55 , la figure 5 à la résolution 225. Les lignes droites sur les parties de ces figures correspondant à la direction $y$ sont les limitations à $\pm 2 \mathrm{~mm}\left(0,3 r_{0}\right)$ introduites par le trou d'entrée. Les courbes tracées sont les aires théoriques d'acceptance à $50 \%$ et à $100 \%$ de trans- 


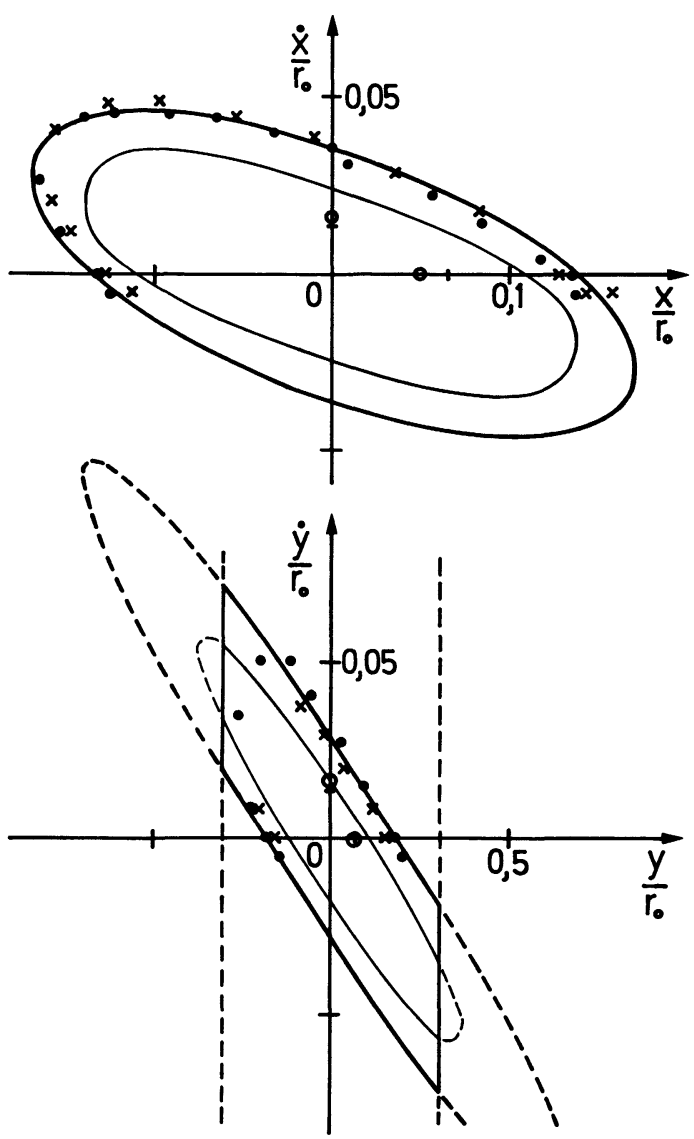

Fig. 4. - Aires d'acceptance à $50 \%$ et $100 \%$ de transmission pour $n=2$ cycles HF et $R=55$. (En haut : direction $x$; en bas : direction $y$. Ellipses théoriques à $50 \%$ : trait fort ; points expérimentaux à $50 \%: \bullet:$ ions $\mathrm{Rb}^{+}$de $20 \mathrm{eV} ; \times$ : ions $\mathrm{Cs}^{+}$de $32 \mathrm{eV}$. Aires théoriques à $100 \%$ : trait fin; limites de Paul et al. [2] : + ; points expérimentaux à $100 \%$ : 0 .)

[Acceptance areas for $50 \%$ and $100 \%$ transmission with $n=2 \mathrm{RF}$ cycles and $R=55$. (Above : $x$ direction; below : $y$ direction. $50 \%$ theoretical ellipses : broad line ; $50 \%$ experimental points : - : $20 \mathrm{eV} \mathrm{Rb}^{+}$ions ; $\times$: $32 \mathrm{eV} \mathrm{Cs}^{+}$ions. $100 \%$ theoretical areas : fine line; limits from Paul and al. [2] : + ; $100 \%$ experimental points : O).]

mission; les figures portent aussi les valeurs limites prévues par Paul et al. [2] :

$$
\left\{\begin{array}{l}
u_{100} \simeq \frac{1}{2} \frac{r_{0}}{\sqrt{R}} \text { pour } \dot{u}=0 \\
\dot{u}_{100} \simeq \frac{3,5}{\pi f} \frac{r_{0}}{l} \sqrt{\frac{2 e E}{m}} \text { pour } u=0
\end{array}\right.
$$

(d'après les formules (3) et (4) et pour $\theta$ petit).

L'accord entre les aires d'acceptance expérimentales à $50 \%$ de transmission et les ellipses théoriques est excellent pour $n=2$ cycles HF ; il devient moins bon, mais reste satisfaisant pour $n=1$ et 0,5 cycle HF. Dans ce dernier cas d'ailleurs, Dawson lui-même reconnaît que les approximations de la théorie deviennent discutables, en raison du couplage des équations différentielles en $x$ et $y$ lors de la traversée du champ d'entrée. Les valeurs limites trouvées
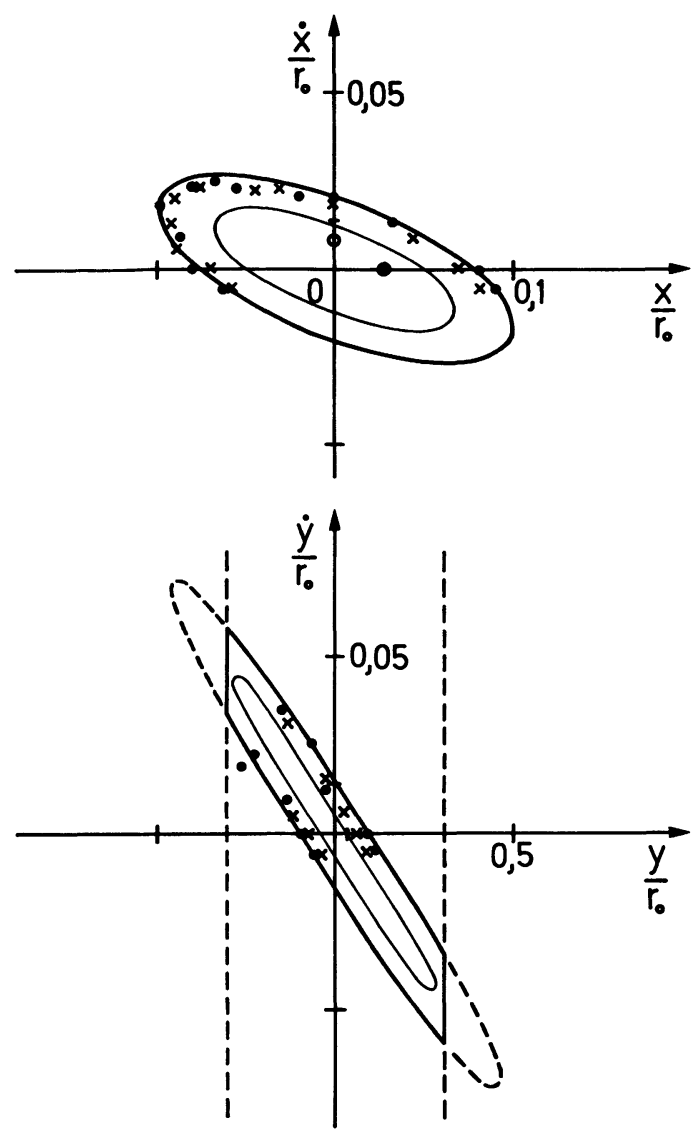

Fig. 5. - Aires d'acceptance à $50 \%$ et $100 \%$ de transmission pour $n=2$ cycles $\mathrm{HF}$ et $R=225$ (autres précisions comme figure 4).

[Acceptance areas for $50 \%$ and $100 \%$ transmission with $n=2 \mathrm{RF}$ cycles and $R=225$ (other precisions as figure 4).]

expérimentalement pour $u_{100}$ et $\dot{u}_{100}$ (voir $\S 4.2$ pour leur obtention) sont en bon accord avec les valeurs de Paul, alors que les aires d'acceptance théoriques à $100 \%$ paraissent un peu grandes.

L'inclinaison des aires d'acceptance correspond à un faisceau incident convergent à l'intérieur du

Tableau II. - Position et taille $d u$ "cross-over" pour $50 \%$ de transmission

[Position and radius of the cross-over for $50 \%$ transmission]

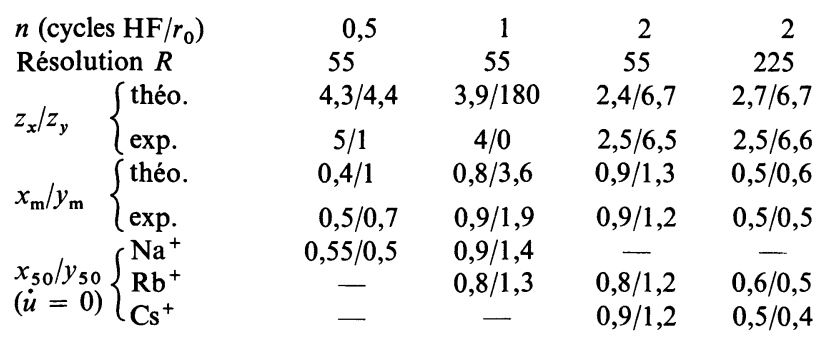

Toutes les cotes sont données en $\mathrm{mm}:$ la précision sur les valeurs expérimentales de $z_{u}\left(z_{x}\right.$ ou $\left.z_{y}\right)$ est de l'ordre de $\pm 1 \mathrm{~mm}$, celle sur $u_{50}$ et $u_{\min }\left(x_{\mathrm{m}}\right.$ ou $\left.y_{\mathrm{m}}\right)$ de l'ordre de $\pm 0,1 \mathrm{~mm}$.

[All dimensions are given in $\mathrm{mm}$; the precision on experimental values of $z_{u}\left(z_{x}\right.$ or $\left.z_{y}\right)$ is ca. $\pm 1 \mathrm{~mm}$, that on $u_{50}$ and $u_{\min }\left(x_{\mathrm{m}}\right.$ or $\left.y_{\mathrm{m}}\right)$ ca. $\pm 0.1 \mathrm{~mm}$.] 
quadrupôle. Dans chacun des plans $x z$ ou $y z$, le faisceau des prolongements des trajectoires transmises à $50 \%$ atteint une largeur minimale $u_{\min }\left(x_{\mathrm{m}}\right.$ ou $\left.y_{\mathrm{m}}\right)$ en un cross-over virtuel situé à l'abscisse $z_{u}\left(z_{x}\right.$ ou $\left.z_{y}\right)$. Le tableau II précise, pour différentes valeurs de $n$, la position $z_{u}$ et la taille $u_{\min }$ de ces cross-overs, et compare les résultats expérimentaux aux prévisions théoriques qu'on peut tirer des calculs de Dawson (voir annexe) :

$$
\begin{aligned}
z_{u} & =\frac{r_{0}}{n \pi} \frac{A}{\Gamma} ; \\
u_{\min } & =r_{0} \sqrt{\frac{\varepsilon}{\Gamma}} .
\end{aligned}
$$

En fait, la position d'un cross-over ne dépend pratiquement que de la valeur de $n$, car les aires d'acceptance à $n$ donné, tracées pour différentes résolutions ou différentes transmissions, sont très sensiblement homothétiques. La taille d'un cross-over est par contre d'autant plus petite que la résolution est plus grande (variation approximative en $1 / \sqrt{R}$ ) ou la transmission plus élevée ; elle coïncide d'ailleurs avec la valeur de $u$ pour laquelle $\dot{u}=0$. Ce point est vérifié, dans le cas de la transmission à $50 \%$, à la dernière ligne du tableau II où figurent les valeurs expérimentales de $u_{50}$ ( $x_{50}$ ou $y_{50}$ ) pour $\dot{u}=0$.

Toutefois, à $n=1$ et 2 cycles HF, le trou d'entrée supprime les extrémités des aires d'acceptance à $50 \%$ dans la direction $y$. A $n=2$ cycles, les trajectoires ainsi éliminées seraient passées à l'intérieur du cross-over, dont la position et la taille ne sont donc pas affectées par cette suppresșion. Par contre, à $n=1$ cycle, ce sont les trajectoires du bord du cross-over qui sont éliminées et c'est le trou d'entrée lui-même qui limite le faisceau. Ceci explique le désaccord apparent à $n=1$ cycle entre la position théorique du cross-over $\left(z_{y}=180 \mathrm{~mm}\right)$ et sa position réelle à l'entrée du champ, ainsi qu'entre la taille théorique $\left(y_{m}=3,6 \mathrm{~mm}\right)$ et la taille effective, forcément inférieure au rayon du trou, soit $2 \mathrm{~mm}$. Cet effet du trou d'entrée s'observe depuis 0,6 cycle jusque vers 1,6 cycle (ces limites correspondent à la condition : $y=2 \mathrm{~mm}$ pour $\dot{y}=0$ ).

Un filtre quadrupolaire reçoit généralement, selon des conditions géométriques identiques, des ions de masses différentes dans une étroite bande d'énergie. Le temps de traversée du champ d'entrée varie d'une espèce ionique à une autre ; il en résulte des cross-overs différents et une discrimination des masses transmises, bien connue expérimentalement [22]. L'acceptance en $x$ (mesurée par $\varepsilon_{x}$ ) est une fonction lentement croissante de $n$, donc de la masse (5), alors que l'acceptance en $y\left(\varepsilon_{y}\right)$ passe par un maximum très aigu vers 1,2 cycle [10]. En disposant un diaphragme à l'entrée du quadrupôle, on peut diminuer l'acceptance en $y$ et en faire une fonction lentement décroissante de $n$ au-delà de 1,5 cycle. On peut ainsi faire en sorte que l'acceptance totale du quadrupôle, dont une estimation est fournie par $\sqrt{\varepsilon_{x} \varepsilon_{y}}$, ne dépende qu'assez peu de la masse des ions sur un intervalle de masse assez étendu (dont la position dépend de l'énergie des ions). Un diaphragme de rayon voisin de $0,7 \mathrm{~mm}$ (soit $0,1 r_{0}$ ) conviendrait pour la résolution 55 , avec une variation de l'acceptance totale inférieure à $10 \%$ entre 0,5 et 2 cycles HF. L'application pratique de cette remarque implique cependant que l'on dispose à l'entrée du quadrupôle une lentille quadrupolaire dénuée d'aberrations, réglée de façon optimale pour les deux directions $x$ et $y$, et ce pour chacune des masses analysées.

4.2 VARIATIONS DE LA TRANSMISSION AVEC LES CONDITIONS D'ENTRÉE. - L'attaque d'un filtre quadrupolaire par un faisceau convergent légèrement à l'intérieur du champ ne saurait qu'être bénéfique. C'est la raison pour laquelle nous avons étudié les variations de la transmission, non seulement dans les cas d'une injection parallèle à l'axe (cas $a$ ) et à partir du point $\mathrm{O}$ de l'axe à l'entrée du champ (cas $b)$, mais

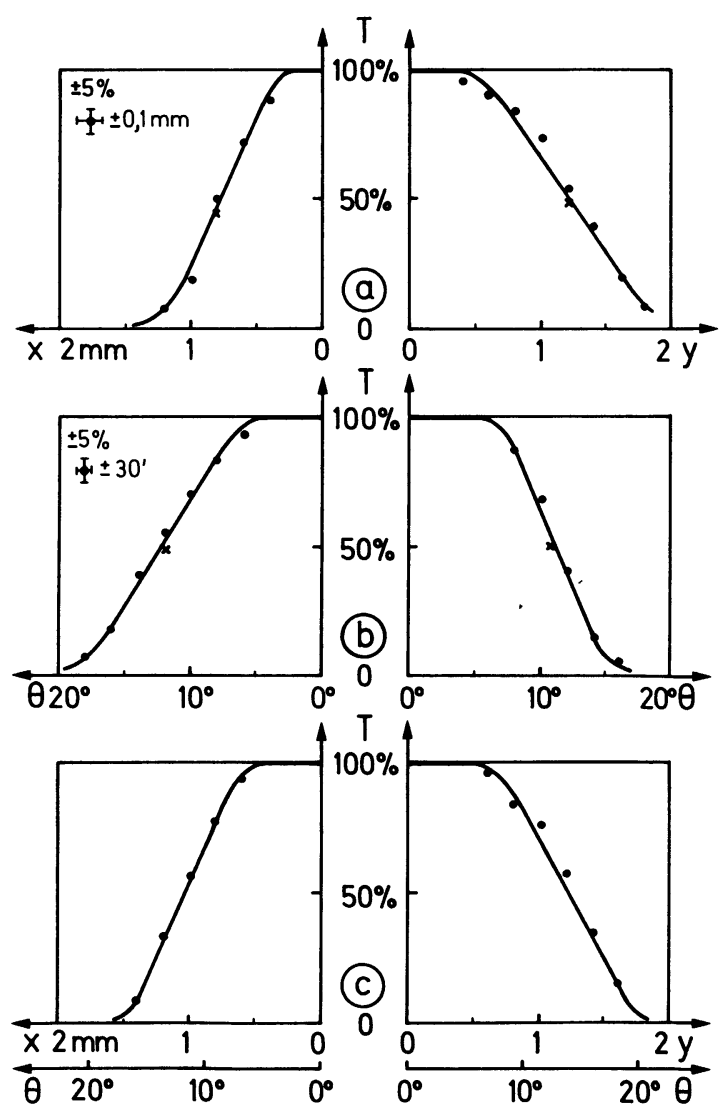

Fig. 6. - Variation de la transmission pour $n=2$ cycles $\mathrm{HF}$ et $R=55: a$ ) en fonction de la distance à l'axe pour une introduction parallèle à l'axe; $b$ ) en fonction de l'inclinaison sur l'axe pour une introduction à partir du point $\mathrm{O} ; c$ ) en fonction de l'inclinaison sur l'axe pour une introduction en direction du point $z=5 \mathrm{~mm}$ $\left(0,75 r_{0}\right)$. (Ions $\mathrm{Rb}^{+}$de $20 \mathrm{eV}$. A gauche : plan $x z$; à droite : plan $y z$.)

[Variations of the transmission for $n=2$ RF cycles and $R=55$ : a) versus the distance to the axis for an introduction parallel to the axis $; b)$ versus the input angle for an introduction from point $\mathrm{O}$; c) versus the input angle for an introduction towards the point $z=5 \mathrm{~mm}\left(0,75 r_{0}\right) .\left(20 \mathrm{eV} \mathrm{Rb^{+ }}\right.$ ions. Left $: x z$ plane ; right $: y z$ plane.)] 
aussi dans le cas d'un ion introduit en direction d'un point de l'axe arbitrairement choisi à l'abscisse $z=5 \mathrm{~mm}$, soit $0,75 r_{0}$ (cas $c$ ). Ce choix correspond approximativement à la position moyenne des crossovers (Tabl. II). Les paramètres $u$ et $\theta$ dans le cas $c$ sont liés par la relation évidente :

$$
u=-0,75 r_{0} \operatorname{tg} \theta \quad \text { (Fig. 1) . }
$$

Toutes les courbes de transmission obtenues, soit en fonction de l'écart à l'axe de la trajectoire initiale (cas $a$ : Fig. $3 a$ et $6 a$ ), soit en fonction de l'inclinaison sur l'axe de cette trajectoire (cas $b$ : Fig. $3 b$ et $6 b$ et cas $c$ : Fig. $6 c$ ), présentent le même aspect en trois parties :

- Un plateau à $100 \%$ de transmission jusqu'à une valeur limite de $u$ (ou de $\theta$ ) notée $u_{100}$ (ou $\theta_{100}$ ). Ce plateau disparaît si la vitesse des ions est trop élevée pour permettre la résolution adoptée avec $100 \%$ de transmission (c'est-à-dire si $n<n_{\min }$ ); un minimum peut même apparaître sur l'axe [23].

- Une partie à décroissance rapide et pratiquement rectiligne au cours de laquelle la transmission passe à la valeur $50 \%$ pour $u_{50}$ (ou $\theta_{50}$ ). Les valeurs de $u_{100}$ (ou $\theta_{100}$ ) sont définies par l'intersection du prolongement linéaire de cette partie de la courbe avec le plateau à $100 \%$.
- Une queue à décroissance plus lente où la transmission devient faible. Dans cette région qui ne présente guère d'intérêt pratique, les mesures de la transmission peuvent être quelque peu sous-estimées (voir § 3.2).

Les données essentielles des courbes de transmission portent donc sur les valeurs de $u_{100}$ et $u_{50}$ (ou $\theta_{100}$ et $\left.\theta_{50}\right)$. Le tableau III rassemble les résultats obtenus par les plans $x z(\varphi=0)$ et $y z(\varphi=\pi / 2)$ et les compare aux prévisions théoriques de Paul et al. [2] et de Dawson [10] (voir schéma explicatif à la figure 7). On trouvera plus loin, au $\S 4.3$, l'étude de l'évolution de la transmission dans les plans intermédiaires $(0<\varphi<\pi / 2)$.

De l'examen du tableau III, et plus généralement des courbes de transmission, on peut dégager les conclusions suivantes :

- Pour des conditions d'entrée données (cas $a, b$ ou $c$ ), la transmission ne fait intervenir que la vitesse des ions (à fréquence HF imposée) et la résolution adoptée. C'est ainsi que les résultats obtenus pour les mêmes valeurs de $n$ et de $R$ - c'est-à-dire aux colonnes 3 et 4,6 et 8,7 et $9-$ sont identiques, à la précision des mesures.

- Il n'est pas possible d'atteindre la résolution de $100 \%$ si les ions sont trop rapides pour la résolution

Tableau III. - Déplacements u (x ou y) ou angles limites $\theta\left(\theta^{x}\right.$ ou $\left.\theta^{y}\right)$ pour les transmissions à $100 \%$ et $50 \%$ [Displacements $u(x$ or $y)$ or limiting angles $\theta\left(\theta^{x}\right.$ or $\left.\theta^{y}\right)$ for $100 \%$ and $50 \%$ transmissions]

\begin{tabular}{|c|c|c|c|c|c|c|c|c|c|c|}
\hline \multicolumn{2}{|c|}{ Nature des ions } & $\mathrm{Na}^{+}$ & $\mathrm{Na}^{+}$ & $\mathrm{Na}^{+}$ & $\mathbf{R b}^{+}$ & $\mathbf{R b}^{+}$ & $\mathbf{R b}^{+}$ & $\mathbf{R b}^{+}$ & $\mathrm{Cs}^{+}$ & $\mathrm{Cs}^{+}$ \\
\hline \multirow{4}{*}{\multicolumn{2}{|c|}{$\begin{array}{l}\text { Energie }(\mathrm{eV}) \\
\left.n \text { (cycles } \mathrm{HF} / r_{0}\right) \\
\text { Résolution } R \\
\text { Cas } a: u_{100} \text { théo. }\end{array}$}} & 100 & 100 & 22 & 81 & 81 & 20 & 20 & 32 & 32 \\
\hline & & 0,5 & 0,5 & 1 & 1 & 1 & 2 & 2 & 2 & 2 \\
\hline & & 20 & 55 & 55 & 55 & 225 & 55 & 225 & 55 & 225 \\
\hline & & 0,6 & $(0,45)$ & 0,45 & 0,45 & $(0,22)$ & 0,45 & 0,22 & 0,45 & 0,22 \\
\hline \multirow{3}{*}{\multicolumn{2}{|c|}{$\begin{array}{ll}x_{100} / y_{100} & \left\{\begin{array}{l}\text { théo. } \\
\text { exp. }\end{array}\right. \\
x_{50} / y_{50} & \left\{\begin{array}{l}\text { théo. } \\
\text { exp. }\end{array}\right.\end{array}$}} & - & $(0,3 / 0,5)$ & $0,35 / 1,8$ & $0,35 / 1,8$ & - & $0,8 / 0,85$ & $0,40 / 0,46$ & $0,8 / 0,85$ & $0,40 / 0,46$ \\
\hline & & $\underline{0,3 / 0,25}$ & $\begin{array}{c}0 / 0 \\
0,4 / 1\end{array}$ & $\begin{array}{c}0,35 / 0,5 \\
0,8 / 3,6\end{array}$ & $\begin{array}{l}0,3 / 0,4 \\
0,8 / 3,6\end{array}$ & $\underline{0 / 0}$ & $\begin{array}{c}0,35 / 0,45 \\
0,9 / 1,3\end{array}$ & $\begin{array}{l}0,2 / 0,2 \\
0,5 / 0,6\end{array}$ & $\begin{array}{c}0,35 / 0,4 \\
0,9 / 1,3\end{array}$ & $\begin{array}{l}0,2 / 0,2 \\
0,5 / 0,6\end{array}$ \\
\hline & & $1,2 / 0,95$ & $0,55 / 0,5$ & $0,9 / 1,4$ & $0,8 / 1,3$ & $0,15 / 0,4$ & $0,80 / 1,2$ & $0,6 / 0,5$ & $0,9 / 1,2$ & $0,5 / 0,4$ \\
\hline \multirow{2}{*}{$\theta_{100}^{x} / \theta_{100}^{y}$} & \{théo. & - & $\left(2^{\circ} 20 / 2^{\circ} 20\right)$ & $40 / 5^{\circ}$ & $4 \% / 5^{\circ}$ & - & $9^{\circ} 20 / 6^{\circ} 50$ & $5^{\circ} / 3^{\circ} 20$ & $9^{\circ} 20 / 6^{\circ} 50$ & $5^{\circ} / 3^{\circ} 20$ \\
\hline & $\begin{array}{l}\text { exp. } \\
\text { théo. }\end{array}$ & $2^{\circ / 1}{ }^{\circ}$ & $\begin{array}{c}0 / 0 \\
3^{\circ} 40 / 3^{\circ} 40\end{array}$ & $\begin{array}{c}2^{\circ} 30 / 1^{\circ} 40 \\
8^{\circ} 40 / 7^{\circ}\end{array}$ & $\begin{array}{c}2^{\circ} 20 / 1^{\circ} 20 \\
8^{\circ} 40 / 7^{\circ}\end{array}$ & $0 / 0$ & 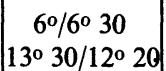 & $\begin{array}{c}2^{\circ} / 2^{\circ} \\
7^{\circ} 10 / 4^{\circ}\end{array}$ & 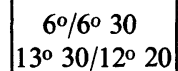 & $\begin{array}{c}2^{\circ} / 2^{\circ} \\
7^{\circ} 10 / 4^{\circ}\end{array}$ \\
\hline $\begin{array}{l}\theta_{50}^{x} / \theta_{50}^{y} \\
\text { Cas } c\end{array}$ & $\left\{\begin{array}{l}\text { exp. } \\
\text { exp. }\end{array}\right.$ & $8^{\circ} 20 / 5^{\circ}$ & $4^{\circ} 20 / 2^{\circ}$ & $10^{\circ} / 6^{\circ}$ & $90 / 5^{\circ} 30$ & $2^{\circ} / 1^{\circ} 10$ & $12 \% / 11^{\circ}$ & $7^{\circ} 30 / 3^{\circ} 40$ & $13^{\circ} 30 / 11^{\circ} 30$ & $6^{\circ} 30 / 3^{\circ} 30$ \\
\hline \multirow{2}{*}{$\theta_{100}^{x} / \theta_{100}^{y}$} & Sthéo. & - & $\left(2^{\circ} 30 / 2^{\circ} 10\right)$ & $5^{\circ} 20 / 5^{\circ} 20$ & $5^{\circ} 20 / 5^{\circ} 20$ & - & $9 \% / 14^{\circ} 30$ & $5^{\circ} 20 / 6^{\circ} 30$ & $9^{\circ} / 14^{\circ} 30$ & $5^{\circ} 20 / 6^{\circ} 30$ \\
\hline & $\begin{array}{l}\text { exp. } \\
\text { théo. }\end{array}$ & $\begin{array}{c}2^{\circ} 30 / 1^{\circ} 30 \\
-\end{array}$ & $\begin{array}{c}0 / 0 \\
5^{\circ} 10 / 3^{\circ} 30\end{array}$ & $\begin{array}{c}30 / 1^{\circ} 50 \\
90 / 8^{\circ}\end{array}$ & $\begin{array}{c}3^{\circ} 30 / 1^{\circ} 40 \\
9 \% / 8^{\circ}\end{array}$ & $0 / 0$ & 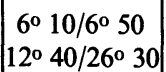 & $\mid \begin{array}{ccc}4^{\circ} & 30 / 5^{\circ} & 20 \\
8^{\circ} & 30 / 1^{\circ} & 30\end{array}$ & 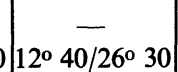 & 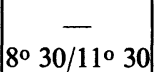 \\
\hline \multirow{2}{*}{$\begin{array}{l}\theta_{50}^{x} / \theta_{50}^{y} \\
x_{50} / y_{50}\end{array}$} & exp. & $10^{\circ} / 4^{\circ} 30$ & $4^{\circ} / 1^{\circ} 40$ & $10^{\circ} / 5^{\circ} 30$ & $9 \% / 6^{\circ} 10$ & $1^{\circ} 40 / 1^{\circ} 20$ & $11^{\circ} 40 / 13^{\circ} 50$ & $10^{\circ} / 11^{\circ} 20$ & - & - \\
\hline & exp. & $0,9 / 0,4$ & $0,4 / 0,15$ & $0,9 / 0,5$ & $0,8 / 0,55$ & $0,15 / 0,15$ & $1 / 1,2$ & $0,9 / 1$ & - & - \\
\hline
\end{tabular}

Cas $a$ : faisceau parallèle à l'axe; cas $b$ : faisceau convergent en $O$; cas $c$ : faisceau convergent en $z=5 \mathrm{~mm}$. A l'exception du eas $a$ où figurent les valeurs $u_{100}$ de Paul et al. (1), toutes les valeurs théoriques de $u$ et $\theta$ sont obtenues à partir des/calculs de Dawson (Fig.7) ; les tirets correspondent à labsence de données ou de mesures, les valeurs entre parenthèses aux cas où les ions sont trop rapides pour la résolution adoptée $\left(n<n_{\min }\right)$.

[Case $a$ : beam parallel to the axis ; case $b$ : beam convergent towards point $O$; case $c$ : beam convergent towards $z=5 \mathrm{~mm}$. With the exception of the case $a$ where are given the $u_{100}$ values from Paul et al. (1), all the theoretical values of $u$ and $\theta$ are derived from Dawson's calculations (Fig. 7); dashes correspond to the absence of theoretical values or measurements, values in parentheses to those cases where the ion energy is too high for the chosen resolution $\left(n<n_{\min }\right)$.] 


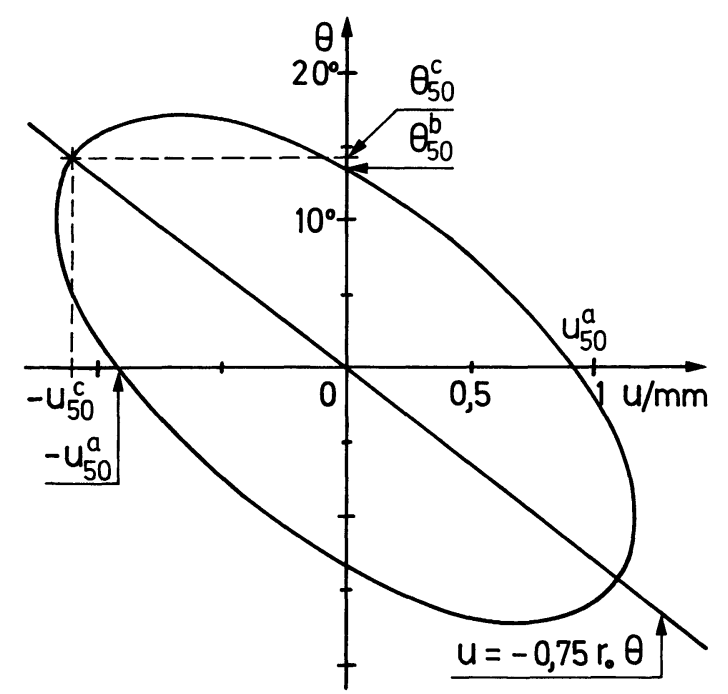

Fig. 7. - Détermination des valeurs théoriques de $u_{50}$ et $\theta_{50}$ à partir des ellipses d'acceptance. (Valeurs numériques pour la direction $x, n=2$ cycles $\mathrm{HF}$ et $R=55$.)

[Determination of the theoretical values of $u_{50}$ and $\theta_{50}$ from acceptance ellipses (numerical values for $x$ direction, $n=2$ RF cycles and $R=55)$.]

adoptée (colonnes 2 et 5), c'est-à-dire si $n$ est inférieur à la valeur minimale $n_{\min }$ donnée au tableau $\mathrm{I}$.

- Les valeurs expérimentales de $u_{100}$ dans le cas $a$ sont en bon accord avec la valeur de Paul (1), alors que les prévisions de Dawson conduisent à des valeurs un peu fortes. L'accord est bien meilleur pour $u_{50}$ (tenu compte de ce que $u<2 \mathrm{~mm}$ à cause du trou d'entrée).

- Contrairement aux prévisions de Paul et al., l'angle limite $\theta_{100}$ dans le cas $b$ n'est pas fixé uniquement par la géométrie du quadrupole (la formule (3) conduit à la valeur $\left.5^{\circ} 20^{\prime}\right)$. Comme le prévoit Dawson, l'angle limite $\theta_{100}$ n'a pas la même valeur dans les plans $x z$ et $y z$; il augmente avec $n$ à $R$ fixée et, à $n$ donné, diminue quand la résolution croît. Là encore, les valeurs théoriques de Dawson sont généralement plus élevées que les mesures, l'accord étant meilleur pour $\theta_{50}$.

- Les valeurs de $\theta_{50}$ et $\theta_{100}$ obtenues dans le cas $c$ (focalisation en $z=5 \mathrm{~mm}$ ) sont pour la plupart à peine différentes de celles obtenues dans le cas $b$ (focalisation en $z=0$ ). Par contre, les valeurs de $u_{50}$ dans le cas $c$ peuvent différer notablement de celles de $u_{50}$ dans le cas $a$ (injection parallèle à l'axe). La direction $y$ semble surtout moins favorisée pour $n=0,5$ et 1 cycle. Ces conclusions sont, bien entendu, conséquences de la position choisie pour le point de focalisation. Comme le montre la figure 7 , la droite

$u=-0,75 r_{0} \operatorname{tg} \theta \simeq-0,75 r_{0} \theta$

est généralement voisine du grand axe de l'ellipse d'acceptance et son intersection avec celle-ci conduit effectivement à des valeurs de $\theta_{50}^{\mathrm{c}}$ proches de $\theta_{50}^{\mathrm{b}}$, alors que $u_{50}^{\mathrm{c}}$ et $u_{50}^{\mathrm{a}}$ peuvent être assez différents l'un de l'autre.
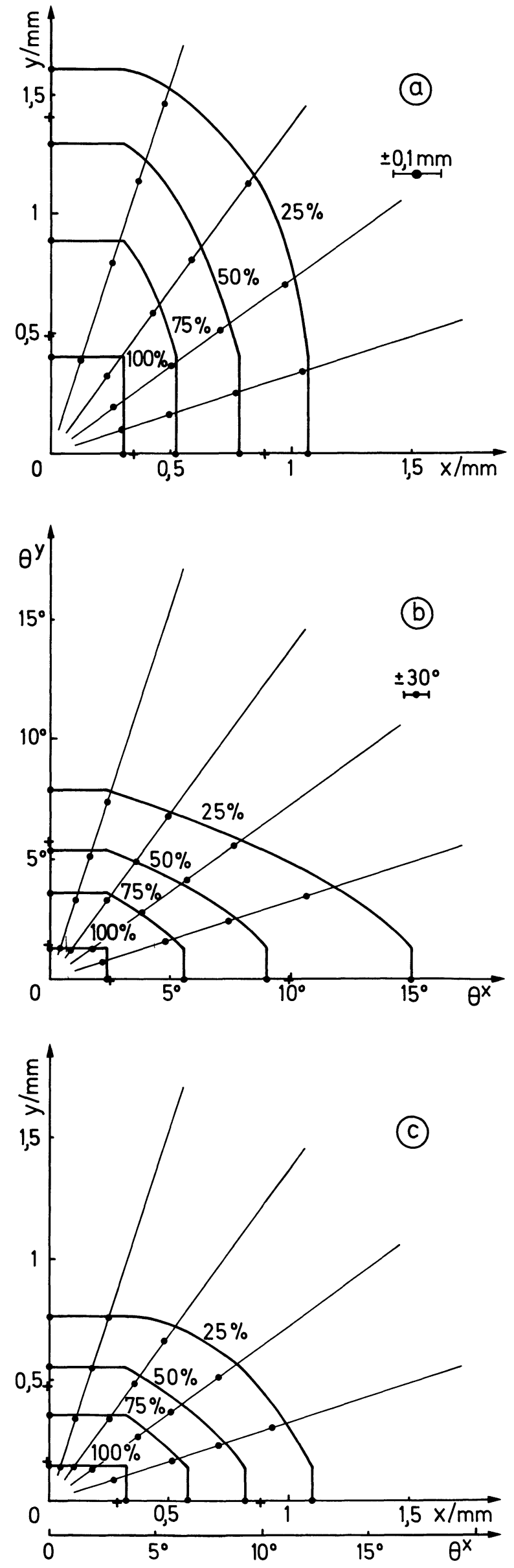
4. 3 Courbes D'ISOTRANSMission. - Les courbes de transmission se déforment progressivement quand on fait varier l'angle azimutal $\varphi$, passant de l'introduction dans le plan $x z(\varphi=0)$ à l'introduction dans le plan $y z(\varphi=\pi / 2)$. La présentation des résultats expérimentaux est, nous semble-t-il, plus parlante dans une représentation polaire $\left(u_{\mathrm{T}}, \varphi\right)\left[\mathrm{ou}\left(\theta_{\mathrm{T}}, \varphi\right)\right]$, où $u_{\mathrm{T}}\left(\mathrm{ou} \theta_{\mathrm{T}}\right)$ est la valeur de $u$ (ou de $\theta$ ) pour laquelle la transmission a une valeur donnée $T$. Les courbes ainsi tracées pour différentes valeurs de $T$ constituent des courbes d'isotransmission, en généralisant une notion introduite par Rüdenauer [4] pour la transmission $100 \%$. Lorsque la direction d'injection des ions ne passe pas par le point $\mathrm{O}$ (cas $a$ ou $c$ par exemple), les courbes d'isotransmission limitent les diverses régions du plan $z=0$ normal à l'axe, à l'intérieur desquelles doivent passer les ions pour qu'ils soient transmis avec une probabilité au moins égale à $T$, quelle que soit la phase du champ HF à leur entrée dans le champ. Dans le cas d'une injection en $\mathrm{O}$ (cas $b$ ), ce sont les valeurs limites des angles d'entrée $\theta$ qui sont portées.

Les résultats obtenus pour la résolution 55 sont portés sur les figures 8 (ions $\mathrm{Rb}^{+}$de $81 \mathrm{eV}$; $n=1$ cycle $\mathrm{HF}$ ) et 9 (ions $\mathrm{Rb}^{+}$de $20 \mathrm{eV}$; $n=2$ cycles HF) dans les trois situations d'introduction des ions déjà envisagées $(a, b, c)$. Les courbes tracées sur ces mêmes figures résultent d'un calcul effectué à partir des transmissions expérimentales $T_{x}$ et $T_{y}$ respectivement dans les directions $x$ et $y$. Ce calcul repose sur les hypothèses suivantes :

- On suppose négligeable le couplage introduit entre les équations différentielles qui décrivent le mouvement d'un ion, soit lors de la traversée du champ d'entrée (ce point a été vérifié par Dawson [10] pour $n>0,5$ cycle HF), soit en raison de l'imperfection de la géométrie du champ quadrupolaire du fait de l'utilisation de barres à section circulaire (cet effet est d'autant plus sensible que l'ion est plus lent et le plan d'injection proche de $\varphi=\pi / 4$ ou $3 \pi / 4$ )

- On admet l'absence de corrélation entre les directions $x$ et $y$ pour les phases initiales qui conduisent à l'élimination des ions par collision sur l'une des barres. Dans le cas $a(\theta=0)$, on écrit donc :

$T(u, \varphi)=T_{x}(x) \times T_{y}(y)$

Fig. 8. - Courbes d'isotransmission pour $n=1$ cycle $\mathrm{HF}$ et $R=55 .\left(+:\right.$ ions $\mathrm{Na}^{+}$de $22 \mathrm{eV} ; \bullet:$ ions $\mathrm{Rb}^{+} \mathrm{de} 81 \mathrm{eV}$. Courbes en trait continu calculées à partir de $T_{x}$ et $T_{y} . \mathrm{a}, \mathrm{b}, \mathrm{c}:$ comme figure 6.)

[Isotransmission curves for $n=1 \mathrm{RF}$ cycle and $R=55$. ( + : $22 \mathrm{eV} \mathrm{Na}^{+}$ions $; \bullet: 81 \mathrm{eV} \mathrm{Rb}^{+}$ions. Solid curves calculated from $T_{x}$ and $T_{y} \cdot \mathrm{a}, \mathrm{b}, \mathrm{c}:$ as figure 6).]

Fig. 9. - Courbes d'isotransmission pour $n=2$ cycles $\mathrm{HF}$ et $R=55$. (๑ : ions $\mathrm{Rb}^{+}$de $20 \mathrm{eV} ; \times$ : ions $\mathrm{Cs}^{+}$de $32 \mathrm{eV}$. Courbes en trait continu calculées à partir de $T_{x}$ et $T_{y} . \mathrm{a}, \mathrm{b}, \mathrm{c}:$ comme figure 6.) [Isotransmission curves for $n=2 \mathrm{RF}$ cycles and $R=55$. ( $\bullet$ : $20 \mathrm{eV} \mathrm{Rb}^{+}$ions ; $\times: 32 \mathrm{eV} \mathrm{Cs}^{+}$ions. Solid curves calculated from $T_{x}$ and $T_{y} . \mathrm{a}, \mathrm{b}, \mathrm{c}:$ as figure 6).]
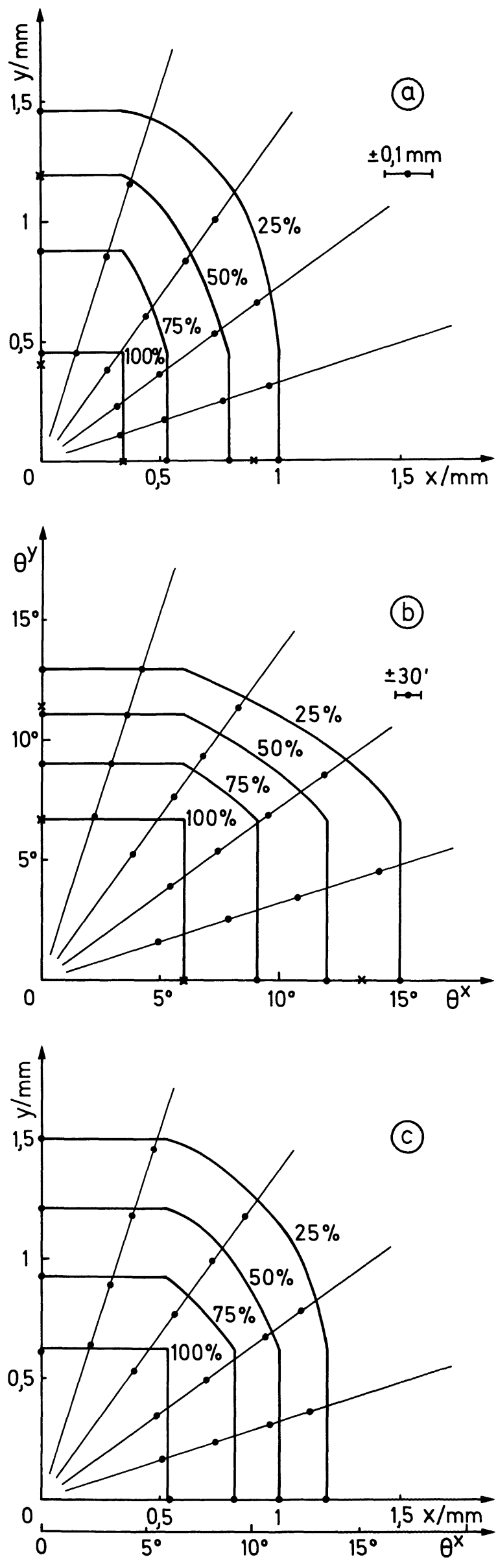
où $x$ et $y$ sont les projections sur $\mathrm{O} x$ et $\mathrm{O} y$ du vecteur $(u, \varphi)$. Dans les cas $b(u=0)$ et $c\left(u=-0,75 r_{0} \operatorname{tg} \theta\right)$, on a :

$T(\theta, \varphi)=T_{x}\left(\theta_{x}\right) \times T_{y}\left(\theta_{y}\right)$

où $\theta_{x}$ et $\theta_{y}$ sont les angles d'entrée des trajectoires projections sur les plans $x z$ et $y z$ de la trajectoire initiale définie par $(\theta, \varphi)$; ces angles sont donnés par :

$\left\{\begin{array}{l}\operatorname{tg} \theta_{x}=\operatorname{tg} \theta \cos \varphi \\ \operatorname{tg} \theta_{y}=\operatorname{tg} \theta \sin \varphi .\end{array}\right.$

L'hypothèse d'absence de corrélation entre les phases éliminées dans les directions $x$ et $y$ est certes parfaitement arbitraire, mais, outre que les courbes d'isotransmission ainsi calculées sont en assez bon accord avec les résultats expérimentaux, elle peut se justifier de la façon suivante. Tant que la transmission n'est pas trop basse $(T>50 \%)$, les phases initiales effectivement éliminées ne sont pas les mêmes en $x$ et en $y$ : à $n=2$ cycles par exemple, et pour $\theta=0$ (cas $a$ ), l'élimination se produit autour de $\pi / 2$ en $x$ et autour de 0 en $y$; à $n=4,5$ cycles, elle se produit autour de $\pi$ en $x$ et autour de 0 en $y$ [7]. Une hypothèse d'exclusion mutuelle des phases éliminées conduirait à :

$T(u, \varphi)=1-\left(1-T_{x}\right)-\left(1-T_{y}\right)=T_{x}(x)+T_{y}(y)-1$

formule qui, aux transmissions élevées, conduit à des valeurs de la transmission très proches de celles données par la formule (8), mais qui fournit des valeurs beaucoup trop petites, rapidement nulles, aux faibles transmissions. Ceci provient de ce que la probabilité de coïncidence des phases éliminées dans les deux directions, négligeable aux fortes transmissions, devient très importante aux faibles transmissions; elle est alors mieux prise en compte dans les formules $(8)$ et $\left(8^{\prime}\right)$.

Les aires d'isotransmission à $100 \%$ devraient être des rectangles limités par $x_{100}$ et $y_{100}$ (ou $\theta_{100}^{x}$ et $\theta_{100}^{y}$ ). Dans les trois cas d'introduction, on obtient en fait un angle arrondi qui traduit peut-être le caractère non strictement quadrupolaire du champ électrique entre les barres, effet d'autant plus marqué que l'ion est plus éloigné de l'axe $\mathrm{O} z$. La transmission n'est donc nullement améliorée pour $\varphi \simeq \pi / 4$, alors qu'un critère d'élimination moins strict que la limitation à $r_{0}$ de l'écart maximal à l'axe a été envisagé dans ce cas [12]. En fait, en dehors des plans $x z$ et $y z$, la trajectoire réelle d'un ion dans le champ quadrupolaire est très loin d'être plane $[2,6]$ et il suffit pratiquement que $x$ ou $y$ atteigne $r_{0}$ pour que l'ion soit éliminé.

Cet arrondi aux valeurs intermédiaires de $\varphi$ se retrouve sur tous les résultats expérimentaux. Peu marqué dans le cas $a$, il est plus accentué dans les cas $b$ et $c$ à $n=2$ cycles, soit parce que la corrélation entre les phases éliminées y est plus marquée, soit parce que ce sont justement les situations où l'ion peut s'écarter plus loin de l'axe et donc subir plus fortement le caractère non strictement quadrupolaire du champ. Comme il se doit, le cas $c$ réalise un certain compromis à la transmission $50 \%$, à la fois entre les directions $x$ et $y$ et, à un moindre degré, entre les temps de traversée 1 et 2 cycles HF.

5. Conclusion. - Cette étude expérimentale de la transmission d'un filtre quadrupolaire en fonction des conditions d'entrée des ions, et la confrontation des résultats obtenus aux théories existantes, confirment que :

- Pour une direction d'injection donnée, la transmission d'un ion dépend essentiellement de la résolution adoptée (c'est-à-dire du rapport $U / V$ des tensions continue et alternative appliquées aux barres) et du nombre $n$ de cycles HF subis par l'ion dans le champ d'entrée du quadrupôle (paramètre qui fait intervenir la vitesse de l'ion, le rayon $r_{0}$ du champ et la fréquence $f$ d'excitation HF).

- L'acceptance d'un filtre quadrupolaire est optimale pour $n$ compris entre 1 et 2 cycles HF ; comme $r_{0}$ et $f$ sont imposés, il serait souhaitable d'adapter l'énergie des ions à leur masse pour satisfaire cette condition.

- L'attaque d'un filtre quadrupolaire doit s'effectuer préférentiellement avec un faisceau d'ions convergent en un cross-over virtuel situé à une distance de l'entrée comprise entre $0,5 r_{0}$ et $r_{0}$; la taille de ce crossover doit être d'autant plus petite que la résolution cherchée est plus grande.

Il est clair toutefois que la grandeur intéressante dans la mise en œuvre d'un filtre quadrupolaire est, non pas la transmission de telle ou telle trajectoire ionique particulière, mais plutôt la transmission globale d'un faisceau plus ou moins largement ouvert, provenant d'une aire émissive relativement étendue, et ayant traversé, avant de pénétrer dans le filtre, divers montages optiques (lentilles de focalisation et éventuellement filtre d'énergies). Ce problème, déjà abordé théoriquement par Dawson [15], fera l'objet de la deuxième partie de cette étude.

Annexe. - Position et taille théoriques du crossover. - Pour des conditions de fonctionnement déterminées, un filtre quadrupolaire est caractérisé, pour chacune des directions $x$ ou $y$, par une ellipse d'acceptance d'équation :

$\Gamma\left(\frac{u}{r_{0}}\right)^{2}+2 A\left(\frac{u}{r_{0}}\right)\left(\frac{\dot{u}}{r_{0}}\right)+B\left(\frac{\dot{u}}{r_{0}}\right)^{2}=\varepsilon$

où $A, B, \Gamma$ et $\varepsilon$ dépendent de la résolution adoptée et du temps de séjour des ions dans le champ de fuite, supposé de longueur $r_{0}$. Introduisant l'angle d'entrée $\theta=n \pi u / r_{0}$ (formule $4^{\prime}$ pour $\theta$ petit), l'équation de l'ellipse s'écrit :

$\Gamma^{\prime} u^{2}+2 A^{\prime} u \theta+B^{\prime} \theta^{2}=\varepsilon$ 
en posant pour la commodité du calcul : $A^{\prime}=A / n \pi r_{0}$, $B^{\prime}=B / n^{2} \pi^{2}$ et $\Gamma^{\prime}=\Gamma / r_{0}^{2}$.

Le prolongement, à l'intérieur du quadrupôle, d'une trajectoire initialement définie par $u$ et $\theta$ (en $z=0$ ), a pour équation :

$u(z)=u+z \theta$.

Le rayon à une abscisse $z$ donnée du faisceau virtuel de trajectoires défini par l'ellipse d'acceptance est égal au maximum de $u(z)$ lorsque $u$ et $\theta$ sont liés par (9), soit $u_{\max }(z)$. Pour le déterminer, on différencie (9) et (10) à $z$ constant :

$\left\{\begin{array}{l}\mathrm{d} u(z)=\mathrm{d} u+z \mathrm{~d} \theta=0 \\ \left(\Gamma^{\prime} u+A^{\prime} \theta\right) \mathrm{d} u+\left(A^{\prime} u+B^{\prime} \theta\right) \mathrm{d} \theta=0\end{array}\right.$

ce qui suppose :

$\left(A^{\prime}-\Gamma^{\prime} z\right) u+\left(B^{\prime}-A^{\prime} z\right) \theta=0$.

Tenu compte de (9) et admettant que $z \neq B^{\prime} / A^{\prime}$, on aboutit à la valeur initiale de $u$, notée $u_{0}(z)$, pour laquelle $u(z)$ est maximal à l'abscisse $z$ considérée :

$u_{0}(z)=\frac{\left|B^{\prime}-A^{\prime} z\right| \sqrt{\varepsilon}}{\sqrt{B^{\prime} \Gamma^{\prime}-A^{\prime 2}} \sqrt{\Gamma^{\prime} z^{2}-2 A^{\prime} z+B^{\prime}}}$.
On en déduit $u_{\max }(z)$ par :

$u_{\max }(z)=u_{0}(z)-z \frac{A^{\prime}-\Gamma^{\prime} z}{B^{\prime}-A^{\prime} z} u_{0}(z)$

soit :

$u_{\max }(z)=\sqrt{\varepsilon} \sqrt{\frac{\Gamma^{\prime} z^{2}-2 A^{\prime} z+B^{\prime}}{B^{\prime} \Gamma^{\prime}-A^{\prime 2}}}$.

La position du cross-over est définie par la valeur $z_{u}$ de $z$ pour laquelle $u_{\max }(z)$ est minimal quand $z$ varie. Dérivant (11) par rapport à $z$ et annulant cette dérivée, on trouve :

$z_{u}=\frac{A^{\prime}}{\Gamma^{\prime}}=\frac{r_{0}}{n \pi} \frac{A}{\Gamma}$

valeur de $z$ pour laquelle $u_{\max }(z)$ a sa valeur minimale $u_{\min }$ telle que :

$u_{\min }=\sqrt{\frac{\varepsilon}{\Gamma^{\prime}}}=r_{0} \sqrt{\frac{\varepsilon}{\Gamma}}$

Le résultat $u_{\min }$ coïncide avec la valeur de $u$ pour laquelle $\theta=0$. Ce sont donc les trajectoires ioniques arrivant parallèlement à l'axe du quadrupôle qui fixent la taille du cross-over. Celui-ci est virtuel, car $z_{u}>0$ correspond à un point situé à l'intérieur du champ quadrupolaire.

\section{Bibliographie}

[1] Paul, W. et Steinwedel, H., Z. Naturforsch. 8a (1953) 448.

[2] Paul, W., Reinhard, H. P. et von Zahn, U., Z. Phys. 152 (1958) 143.

[3] Benninghoven, A. et Loebach, E., Rev. Sci. Instrum. 42 (1971) 49.

[4] Ruedenauer, F. G., Vacuum 22 (1972) 609.

[5] Slodzian, G., Surf. Sci. 48 (1975) 161.

[6] Dawson, P. H., Quadrupole Mass Spectrometry and its Applications (Elsevier Scientific Publishing Company, Amsterdam) 1976.

[7] Dawson, P. H., Int. J. Mass. Spectrom. Ion Phys. 6 (1971) 33.

[8] Dawson, P. H., Int. J. Mass Spectrom. Ion Phys. 14 (1974) 317.

[9] Baril, M. et Septier, A., Revue Phys. Appl. 9 (1974) 525.

[10] Dawson, P. H., Int. J. Mass Spectrom. Ion Phys. 17 (1975) 423.

[11] Ghosh, P. K. et Arora, A. S., Int. J. Mass Spectrom. Ion Phys. 21 (1976) 343.

[12] Ghosh, P. K., Jain, A. et Nagarajan, R., Int. J. Mass Spectrom. Ion Phys. 18 (1975) 1.
[13] Kondo, T., Omura, I. et Hayashi, T., Japan. J. Appl. Phys. 6 (1967) 1137.

[14] Holme, A. E., Thatcher, W. J. et Leck, J. H., J. Phys. E 5 (1972) 429.

[15] Dawson, P. H., Int. J. Mass Spectrom. Ion Phys. 17 (1975) 447.

[16] Weber, R. E. et Cordes, L. F., Rev. Sci. Instrum. 37 (1966) 112.

[17] SePtier, A., Focusing of Charged Particles (Academic Press, New York), II (1967) 123.

[18] Feeney, R. K., Sayle, W. E., II et Hooper, J. W., Rev. Sci. Instrum. 47 (1976) 964.

[19] Arnold, W., J. Vac. Sci. Technol. 7 (1970) 191.

[20] Dawson, P. H., Int. J. Mass Spectrom. Ion Phys. 21 (1976) 317.

[21] Hennequin, J.-F. et Inglebert, R.-L., Int. J. Mass Spectrom. Ion Phys. 26 (1978) 131.

[22] Ehlert, T. C., J. Phys. E 3 (1970) 237.

[23] Offermann, D. et von Zahn, U., Forschungsberichte des Landes Nordrheim-Westfalen $\mathrm{n}^{\mathrm{o}} 1400$ (1964). 